\title{
Adaptive-Multi Parameter MAC Protocol for Reliable Communication in the Smart Grid Environment
}

\author{
Ruqiah Fallatah $^{1}$, Etimad Fadel ${ }^{1}$, Laila Nassef ${ }^{1}$ \\ Computer Science Department, Faculty of Computer and Information Technology, King Abdulaziz University, \\ Jeddah, Kingdom of Saudi Arabia ${ }^{1}$
}

\begin{abstract}
The wide range of smart grid applications rely on wireless sensor networks to monitor and control the smart grid. Each one of these applications has their own quality of service requirements that should be met by these sensor networks. The different communication technologies share the same spectrum band that is used by wireless sensor networks which, may interfere with it and cause network performance degradation. Thus, there is a need to automatically adapt to connectivity changes induced by wireless communications in the smart grid environment. This paper proposes a new adaptive-multi parameter MAC protocol to achieve reliable communications. Three performance parameters of channel quality, packet delivery ratio, and average remaining energy are combined using a weight cost function to adapt the back off time to dynamically select the best communication channels. The proposed MAC protocol is simulated using the MiXiM simulator- based OMNET++ platform to evaluate the network performance. The results indicate the effectiveness of the proposed MAC protocol to mitigate the interference and satisfy the quality of service requirements of the diverse smart grid applications. The results show that the proposed protocol has improved the network performance of about of $25 \%$ increase in successful transmission with lower delay and less energy consumption compared to the basic standard protocol.
\end{abstract}

Keywords: Smart grid, wireless sensor networks, MAC, reliability, IEEE802.15.4.

\section{INTRODUCTION}

There is wide range of applications for Wireless Sensor Networks (WSNs) in smart grid system [1]. Each application has its own Quality of Service (QoS) requirements that must be guaranteed [2]. QoS depends highly on the reliability of communication channels, which varies significantly from channel to channel, and over time. Different technologies, such as IEEE 802.11 (Wi-Fi), and IEEE 802.15.4 (ZigBee) share the unlicensed Industrial, Scientific and Medical (ISM) frequency band [3]. Both IEEE 802.15.4 and IEEE 802.11 systems may be located in the same vicinity (less than 8 meters), in which IEEE802.11 will produce serious interference to IEEE802.15.4 [4]. As the number of wireless devices increases, the communication channels will become unreliable and it cannot guarantee the required QoS for the smart grid applications [2]. The IEEE802.15.4 standard uses a simple blind hopping function [5], where all the channels are scanned and can be uniformly selected at the deployments phase. Then when transmissions suffer from interference on channels, nothing can be done. These factors will result in transmission failures due to loss of either data packet or control packet. This transmission failure will degrade the overall network performance. Since IEEE 802.11 has a wider Radio Frequency (RF) spectrum than IEEE802.15.4 (22 MHz as compared with $3 \mathrm{MHz}$ ), then a single IEEE 802.11 channel can simultaneously cause interference on four adjacent channels [6]. When an IEEE802.15.4 node, which has transmission power about $0 \mathrm{dBm}$, is located within the transmission range of an IEEE 802.11 node, that has much higher power of about 20dBm, this leads to a high impact on IEEE802.15.4 node's communications capabilities [7-9].

The basic IEEE 802.15.4 standard defines the physical (PHY) and Medium Access Control (MAC) layers functionalities for data communication among wireless sensors $[10,11]$. The IEEE 802.15.4 standard operates in two different modes. The beacon-enabled and non-beacon-enabled modes. The period between two beacons is split into an active period and inactive period based on the duty cycle. The active part is again split into two parts, the Contention Access Period (CAP) where nodes compete for channel access using CSMA/CA. The Contention Free Period (CFP) allows the Coordinator to guarantee maximum seven time slots one per device. The Super-frame Duration is the sum of CAP, GTS, and beacon excluding inactive period. Beacon period is the time gap between two successive beacons [12]. This paper considers beacon-enabled mode, where only the CAP period with CSMA/CA are used for communications as shown in Fig.1. 


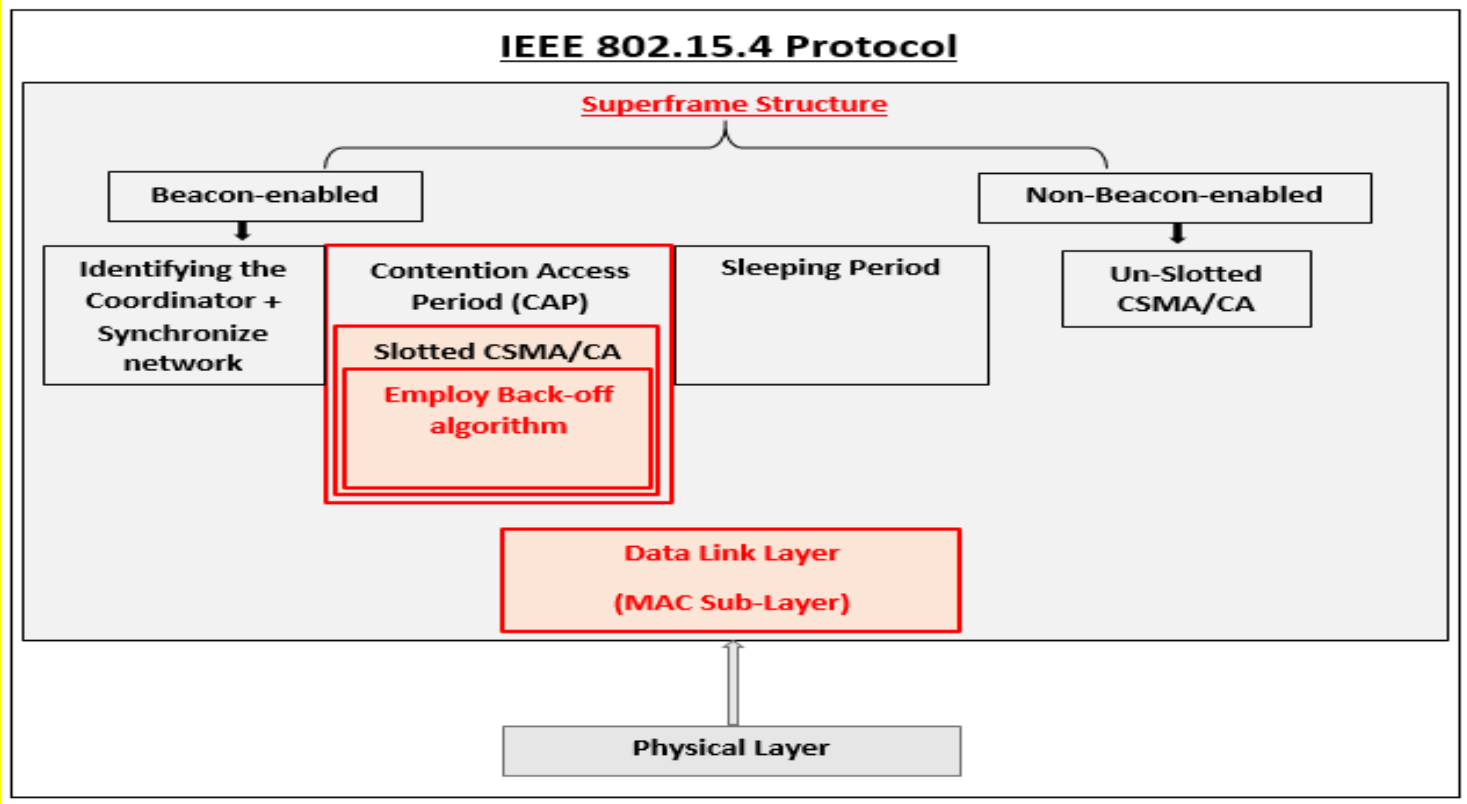

Fig. 1. IEEE802.15.4 MAC Protocols [13]

The sensor nodes have low data rates up to $256 \mathrm{Kbps}$, short-range between $10 \mathrm{~m}-75 \mathrm{~m}$, low latency, and operate with minimum energy requirements $[14,15]$. These nodes are controlled by one coordinator to communicate with any other node in the network [16]. Sensors nodes that utilize CSMA/CA - CAP beacon enabled mode, sense the channel before sending, if it is idle, they will send their frames. If the channel is busy, the sensor will increase its back off exponent to back off even further [18-20]. The Back off time Exponent (BE) which is the waiting period is counted down by a back off counter (NB). Once the back off counter reaches zero and the channel is clear, the node sends data. In general, every transmitting device in the network sets the BE time and it is usually initialized to 2 depending on the value of the battery life extension MAC attribute defined in the standard [16]. The increasing of BE increases the probability of having more back-off delays before accessing the channel [21]. The device senses the channel before transmitting the data packet. If the channel is busy, the node waits for a period, and then checks again to see if the channel is clear. A node sets its back off counter to an integer number over the interval $[1,2]$ of Contention Window $(\mathrm{CW})$. If the node has reached its maximum number of back-offs, it will report to the upper layers that the transmission has failed. If the packet transmission is finished, the node will wait for an acknowledgment (ACK), and if the ACK is received, the packet is successfully transmitted [22]. The purpose of this paper is to add functionalities to traditional coordinator to enable traditional coordinator to sense, take a decision to avoid channels that experience interference, and switch to a more reliable channel. This Channel Aware Coordinator (CACo) senses the channels and allows sensors to access the available channel. The coordinator periodically senses and collects the required parameters from physical layer to estimate channel quality for a number of transmitted packets over any specific channel and updates the average metric accordingly through the scanning operation.

This paper is organized as follows. Section 2 provides literature review for the IEEE802.15.4 MAC protocol standards. Section 3 proposes Adaptive Multi-parameters MAC protocol. Section 4 presents the simulation models, results, and analysis. Section 5 presents the research conclusion and future works.

\section{INTERFERENCE Mitigation IN IEEE 802.15.4 MAC PROTOCOL}

The interference is usually measured by multiple parameters known as Link Quality Indicators (LQI) such as Clear Channel Assessment (CCA), Energy Detection (ED), Received Signal Strength Indicator (RSSI), and Signal to Interference Noise (SINR). These LQIs are directly-measured from the radio transceiver and cannot provide an accurate estimate of link quality because of some wireless phenomena such as blocking, absorption, reflection, scattering, and diffraction, which affect the accuracy of reading [23]. Therefore, an alternative method to measure LQIs must be introduced. The Packet Delivery Ratio (PDR) parameter represents the ratio between number of received and sent packets as 1 . Hence, if the PDR ration drops below 1 it can be an indicator of interference [24]. Recently, a new version of the IEEE 802.15.4 standard is released: IEEE 802.15.4e, which utilizes a new MAC (IEEE 802.15.4e). It is released with time-slotted communications and channel hopping to avoid unreliable channels to increase the reliability of 
communications. However, this new standard is not available in public and there are no sufficient details as the research is still going on [25]. Meanwhile, the basic IEEE 802.15.4 standard is still used and needs some modifications to overcome unreliable links. Little works have been found in literature to solve interference across various technologies summarized in the following table 1.

TABLE 1 A SUMMARY OF PREVIOUS WORKS

\begin{tabular}{|l|l|l|l|l|}
\hline $\begin{array}{l}\text { Reference \& } \\
\text { Publication } \\
\text { Year }\end{array}$ & $\begin{array}{l}\text { Mitigate } \\
\text { Interference }\end{array}$ & $\begin{array}{l}\text { Guarantee QoS } \\
\text { for WSNs in } \\
\text { SG's } \\
\text { Applications }\end{array}$ & $\begin{array}{l}\text { Using CSMA/CA } \\
\text { Transmission } \\
\text { Mechanism }\end{array}$ & $\begin{array}{l}\text { Using } \\
\text { Simulation } \\
\text { Software for } \\
\text { Evaluation }\end{array}$ \\
\hline$[26], 2019$ & $\checkmark$ & Yes & No & Yes \\
\hline$[27], 2019$ & $\checkmark$ & No & Yes & Yes \\
\hline$[28], 2019$ & $\checkmark$ & No & Yes & No \\
\hline$[29], 2019$ & $\checkmark$ & No & Yes & Yes \\
\hline
\end{tabular}

Furthermore, MAC solutions proposed in literature are not suitable for smart grid and do not take its unique characteristics into considerations [26, 27, 30-32]. This motivated us to develop an adaptive MAC protocol for the smart grid environment that can categorize channels into white and blacklist to select best channel for transmission and adapt back off time to ensure satisfaction of QoS requirements. Therefore, the objective of this paper is to propose an adaptive MAC protocol that utilizes three parameters collected from three layers and combines them together using a weight function to adapt back off time and improve the overall network performance.

\section{III.THE PROPOSED ADAPTIVE MULTI PARAMETERS MAC PROTOCOL}

\section{A. $\quad$ Added Functionalities}

The proposed AM-MAC protocol adds new functionalities to traditional coordinator and will be called Channel Aware Coordinator (CACo). Fig.2 shows the super-frame structure of the proposed AM-MAC. The CACo starts with scanning the 16 channels, to detect an idle channel for transmission. Then, it broadcasts the beacon frame with the channel ID to all sensors to access this channel for transmission. The sensors that use CSMA/CA will also be modified to add the new adaptive back off time to access the chosen channel assigned by the CACo. The following pseudo code 1, describes the proposed AM-MAC protocol.

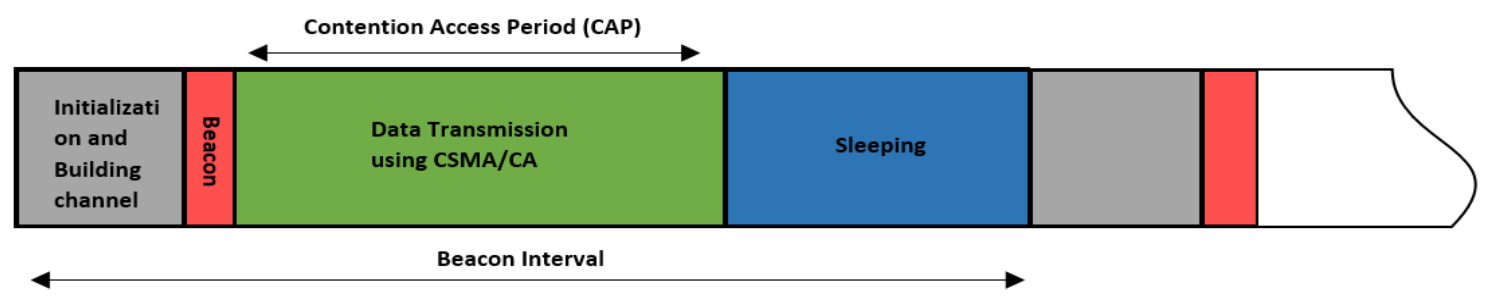

Fig. 2 AM-MAC Protocol Super-Frame Structure

Pseudo code 1: AM-MAC protocol operations

\section{Initialization:}

1. Channel scanning and LQI parameters reported

2. Best channel list building: CACo categorizes the channels that are expected to give better performance as white channel list based on weighting cost function (as will describe in the following subsection A)

\section{Beacon state:}

3. Beacon frame include chosen best channel ID in addition to all information required for transmission, broadcasts to the sensors.

CAP state:

4. If the sensors have data to be transmitted, then 


\section{International Journal of Advanced Research in Computer and Communication Engineering}

Vol. 10, Issue 2, February 2021

DOI 10.17148/IJARCCE.2021.10202

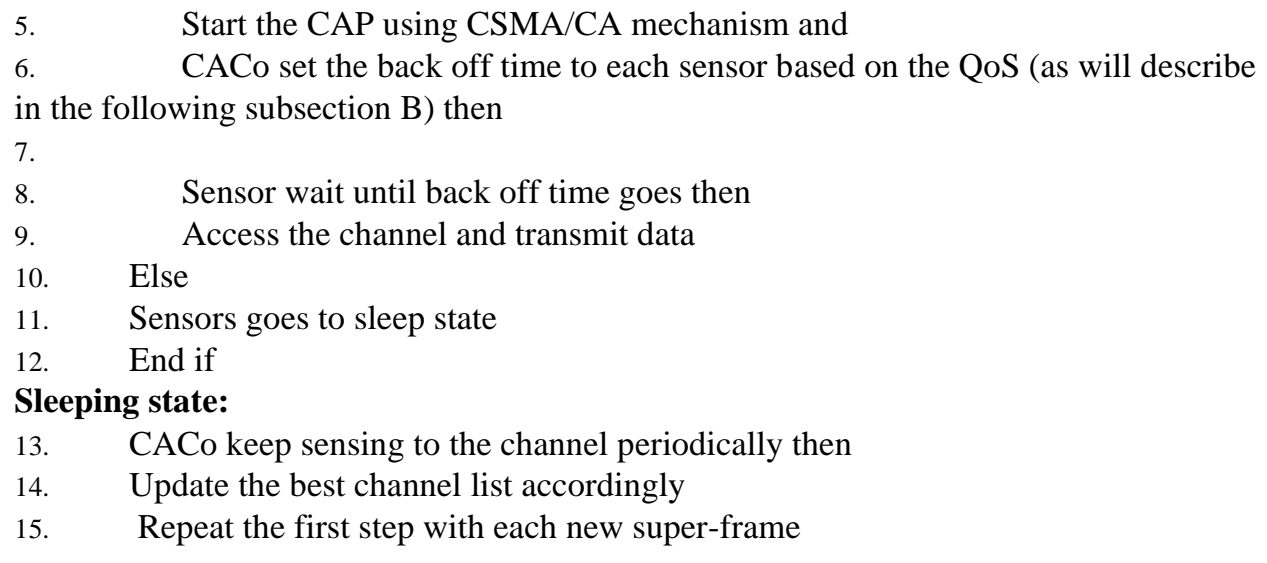

\section{Building the Best Channel List}

The CACo begins execution by building the list of best channels at the beginning of transmission before broadcasting the beacon frame (as listed in step 2 in pseudo code1). The beacon frame will include best channel descriptors in addition, to all other network setup information for example, beacon Interval (BI) time which, includes active and sleeping time of super-frame, and super-frame duration (SD) with the CSMA/CA mechanism time. The CACo broadcasts beacon frame to all sensors in in its cluster. Then it will keep monitoring all channels periodically during sleeping period to define good quality channels. The following algorithm shown in pseudo code 2 describes the processes of building whitelist channels. The CACo adds the channels to whitelist (WL), the list of best channels if their quality is above the predefined threshold. The CACo updates the WL list periodically to build best channels lists that will give satisfactory performance based on the weighting cost function. The first step of building list is to calculate mean and standard deviation of the channel weights using the following equations.

$$
\begin{aligned}
& \mu=\frac{\sum_{k=1}^{C} W A_{k}}{C} \\
& \sigma=\sqrt{\frac{\sum_{k=1}^{C}\left(W A_{k}-\mu\right)^{2}}{C}}
\end{aligned}
$$

Where $\mathrm{C}$ is the channel whitelist vector, WA is average weighting cost.

Then using equations $1 \& 2$ to find the threshold value as indicated in the following equation.

Threshold $=(\mu+\sigma)$

After that, the threshold value is used to create the whitelist channels, where the channels above the threshold value are whitelisted and the channels below the threshold value are blacklisted as shown in equation (4).

$W L=\{c \in C W A \geq$ Threshold $\}$

The WL channels are then sorted in descending order from most reliable to less reliable for dynamic selection of communication channels. Each channel $c \in$ WL will have ID in beacon frame to be broadcast by the CACo. The following pseudo code describes the steps of building whitelist channel list. 


\section{DOI 10.17148/IJARCCE.2021.10202}

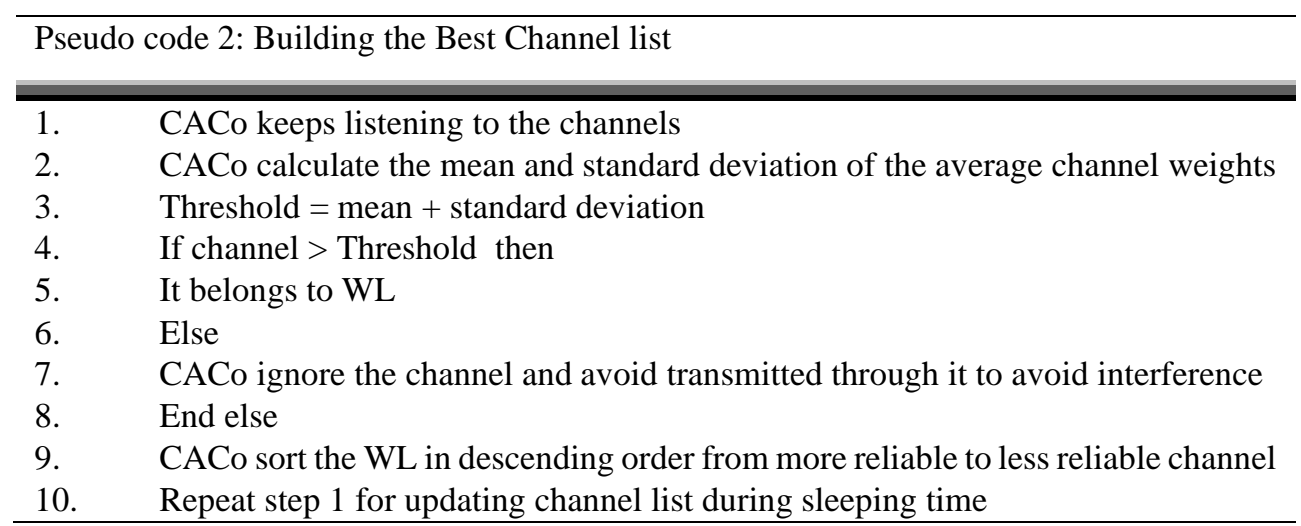

\section{Adapting the Back-off Time}

In AM-MAC, sensors transmit data using CSMA/CA, however the contention window time is not based on how long the node is waiting to send but it varies according to QoS requirements. The CACo can vary size of contention window to allow certain nodes to quickly access the channel with shorter back off time. If required QoS is low, the contention window is big. On the contrary, if QoS is high, the contention window is small. A node that needs to occupy the channel will wait a back off time based on its weight calculation. Thus, sensor node with small weight will have small back off time and will access the channel first. The CACo will broadcast the back off setting parameters to the sensors using the weight function. Fig. 3 shows the process of adapting the back-off time in CSMA/CA, where back-off time is selected from the interval $[0,2 \mu]$ where $(\mu)$ is selected based on Table 2.

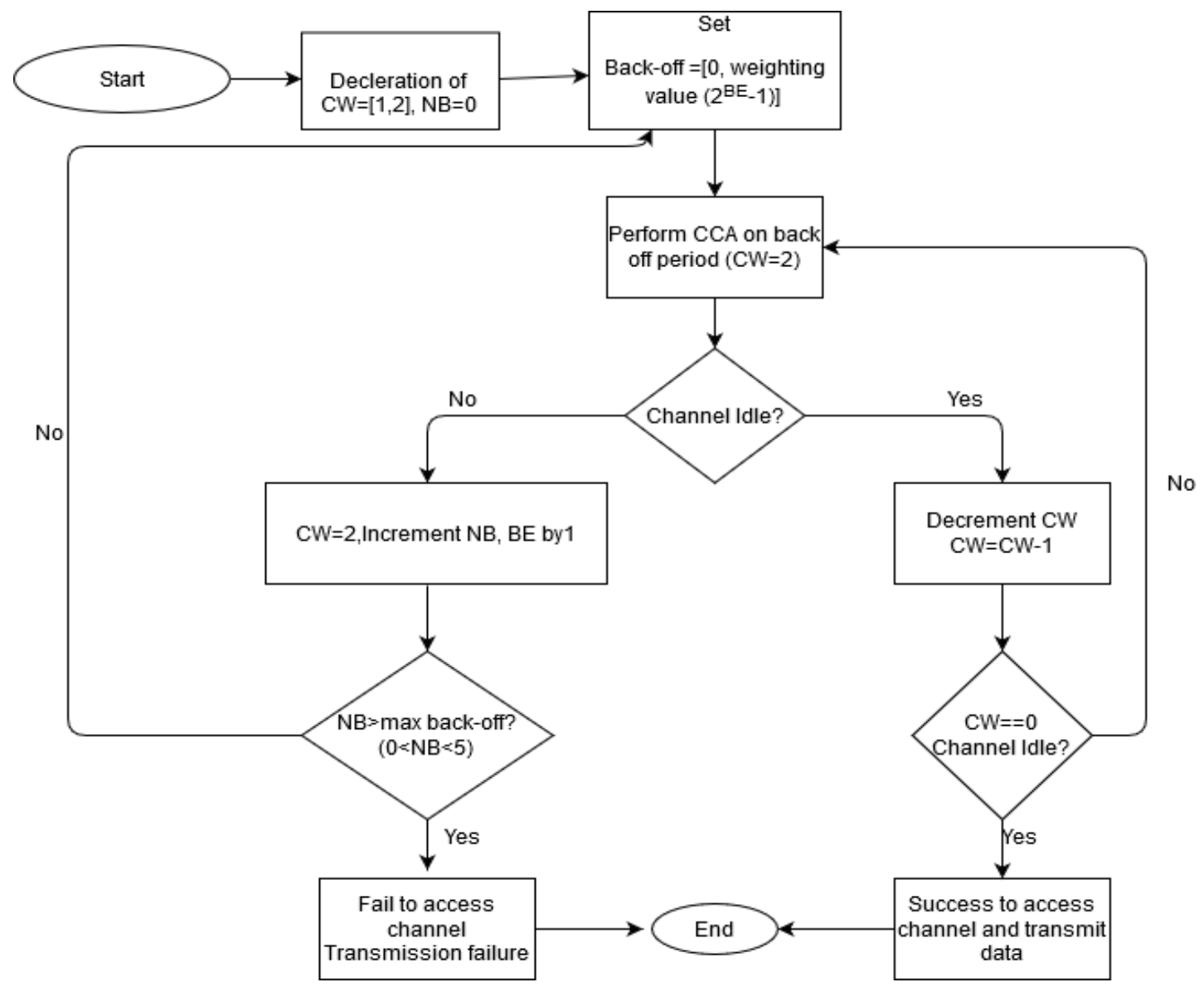

Fig.3 Flowchart of the proposed AM-MAC 


\section{International Journal of Advanced Research in Computer and Communication Engineering}

Vol. 10, Issue 2, February 2021

DOI 10.17148/IJARCCE.2021.10202

\section{B. Weighting Function Parameters}

The weighting function calculates the node's back-off time based on three parameters collected from three different layers to adaptively schedule transmission on the most reliable channel that satisfies required QoS and considers the remaining energy of nodes. The settings of following communications parameters are based on real smart grid communications presented in [3], [30], [31], [33], and [34].

1) Application Layer parameter $\left(W_{Q o S}\right)$

CACo prioritizes data packets generated by application based on their QoS requirement, at the network deployment phase when the network is setup. Data packets are prioritized based on data class, packet size, and data generation rate. Data traffic in the smart grid is classified into three different classes. The first is control, protection and management data of smart grid which have the highest priority (example of this being sudden spike in voltage). Second is the monitored data from different sensors, which have less priority (data about fault locations, temperature, mobility, etc.). The third is the data from smart meters, which have the least priority class (real time pricing data). Examples of SG applications in HANs are the deterministic direct load control that belongs to controlling applications, behavioral energy monitoring that belongs to monitoring applications, and technology enabled dynamic pricing that belongs to smart meter applications. These applications require a data rate $100 \mathrm{kbps}$ and less and have a packet size of 120 or 100 bytes [2]. Each data packet generated is assigned a traffic class value $\left(\mu_{i}\right)$, where the highest data packets are assigned the lowest and ordinary packets are assigned the highest traffic class value. For example, the deterministic direct load control application which is belongs to highest traffic class because of highest QoS requirements will assign to class 1 and so on. Moreover, the data packet that has lower traffic class value (i.e. least priority) will get a small weight and vice versa where $\left(\mathrm{W}_{\mathrm{QoS}}\right)$ is the traffic class value as shown in Table 2 . The weight of traffic class to satisfy QoS $\left(\mathrm{W}_{\mathrm{QoS}}\right)$ is set by the application as mentioned above. The data traffic generated by the sensor traffic is classified into three Sub-classes: class1, class2, and class 3 as presented in Table 2. The classification process is based on the data rate and packet size requirements of different SG applications in the HAN environment to support demand-side applications. The classes' values will prioritize the sensors in accessing the channel. The first class includes control data traffic, which is critical data packets need to transmit to the coordinator within a short time, and high delivery ratio. The second class includes the monitoring data traffic, which is data packets that need to transmit to the coordinator with a high delivery ratio, but no matter the latency. The third class includes the Best-effort smart meter data traffic, which is the data packets only require best-effort support and no matter the packet delivery ratio and latency.

\begin{tabular}{|l|l|}
\hline \multicolumn{2}{|c|}{ TABLE 2 TRAFFIC CLASSIFICATION CLASSES } \\
\hline $\begin{array}{l}\text { Traffic Class Emergency Data Traffic, large packet size, high data } \\
\text { rate. }\end{array}$ & Class Value $\boldsymbol{\mu}_{\tilde{i}}$ \\
\hline Normal Data Traffic, large packets size, no matter data rate. & Class2 \\
\hline $\begin{array}{l}\text { The Best Effort Data Traffic, no matter packets size, data } \\
\text { rate. }\end{array}$ & Class3 \\
\hline
\end{tabular}

Finally, once the data traffic is classified in other words it is given the value $\left(\mu_{i}\right)$, then the value QoS is assigned. The queuing model of priority assignment is used. When a packet arrives at the system, it is added to the queue based on its priority class and every time one packet is selected from the head of the queue that has the highest priority. Network Layer - Packet Delivery Ratio (PDR) parameter:

Similarly, the third parameter $W_{P D R}$ is computed based on PDR from the network layer. The PDR parameter is used to accurately determine the logical channel quality by using the ability of the channel to deliver the data packets successfully. The CACo node continuously monitors transmission from each node independently and the ACKs sent to them. It counts the number of Acknowledgements (ACKs) and the number of packets a particular node sends using equation (5) below. The behavior of each logical channel is computed independently for each device.

$W_{P D R}=\frac{\text { number of Acknowledgements (ACKs) sent }}{\text { number of packets received }}$ 


\section{International Journal of Advanced Research in Computer and Communication Engineering}

Vol. 10, Issue 2, February 2021

DOI 10.17148/IJARCCE.2021.10202

2)

$$
\text { Physical layer Average Remaining Energy }\left(W_{\text {energy }}\right)
$$

The energy required to send data depends on the distance between the nodes and the number of bits which are being transmitted. The energy required for receiving also depends on the number of bits being received. To transmit k-bit of message at distance ' $d$ ' the radio expends energy as shown in equations (6) and (7):

$$
\begin{aligned}
& E T_{x}(k, d)=E T_{x}-\operatorname{elec}(k)+E T_{x}-\operatorname{amp}(k, d) \\
& E T_{x}(k, d)=E e l e c * k+\operatorname{camp}(k, d)
\end{aligned}
$$

Where ETx is the total energy needed to transmit a single k-bit packet to a receiver over a single link of distance d. Eelec is the basic energy for the transmitter; zamp is the multi-path fading coefficient that depends on the transmitter amplifier model.

To receive this message, the radio expends energy as shown in equations (8) and (9):

$$
\begin{aligned}
& E R_{x}(k)=E R_{x}-\operatorname{elec}(k) \\
& E R_{x}(k)=E R_{x}-\text { Eelec } * k
\end{aligned}
$$

Where ERx is the total energy needed to receive a single k-bit packet from a transmitter. Energy consumed by a node Ec is given by equation (10).

$W_{\text {energy }}=E_{i}-E_{r}$

Where, $\mathrm{Ei}=$ Initial Energy of a node, and $\mathrm{Er}=$ Residual Energy of a node. The amount of the energy consumed by nodes will stored in $\left(\mathbf{W}_{\text {energy }}\right)$.

\section{Overall Weighting Function}

The three parameters are combined in one function to adapt the back-off time. The overall weight function $\mu_{\text {overall }}$ depends on the physical layer weight $\left(\mathrm{W}_{\text {energy }}\right)$, network layer weight $\left(\mathrm{W}_{\mathrm{PDR}}\right)$, and application layer weight $\left(\mathrm{W}_{\mathrm{Qos}}\right)$. The proposed overall weighting function, to make the decision to dynamically adapt the back-off time for channel scheduling in MAC layer is defined as follow:

$\mu_{\text {overall }}=\alpha \mathrm{W}_{\mathrm{QoS}}+\beta \mathrm{W}_{\text {energy }}+\gamma \mathrm{W}_{P D R}$

Where the overall weighting value will be then assign to each channel of whitelist and the back off time will be broadcasted to nodes as follow:

Back off $=\left[0,2^{B E}-1\right]$

\section{IV.PERFORMANCE EVALUATION AND RESULTS ANALYSIS}

The Performance of the proposed AM-MAC protocol is evaluated using the OMNET++ based MiXiM network simulator (open-source network simulator was originally designed for WSNs) [33,34]. The proposed protocol is compared with the traditional IEEE 802.15.4 MAC protocol provided in the MiXiM library. The common simulation parameters are listed in the following Table 3. 


\section{International Journal of Advanced Research in Computer and Communication Engineering}

Vol. 10, Issue 2, February 2021

DOI 10.17148/IJARCCE.2021.10202

TABLE 3 THE COMMON SIMULATION PARAMETERS

\begin{tabular}{|l|l|}
\hline Simulation Parameters & Value \\
\hline Number of Sensors & $20 \sim 100$ Sensors in HAN \\
\hline Area & $100 \mathrm{~m}$ x 100m \\
\hline IEEE 802.15 .4 Transmission Range & $30 \mathrm{~m}$ \\
\hline Simulation Time & $300 \mathrm{secs}$ \\
\hline Traffic Type & Constant Bit Rate (CBR) \\
\hline Packets Size & 512 Bytes \\
\hline Propagation Model & Path-loss Shadowing Model / Indoor \\
\hline Path loss exponent & 4.2 \\
\hline Shadowing Variance & 4 \\
\hline Antenna Model & Omni Antenna \\
\hline Radio & CC2420 \\
\hline Data Rate & $250 \mathrm{Kbps}$ \\
\hline Frequencies & $2.4 \mathrm{GHz}$ \\
\hline Rx Current & $17.4 \mathrm{~mA} / \mathrm{bit}$ \\
\hline Tx Current & $19.7 \mathrm{~mA} / \mathrm{bit} / 250 \mathrm{~m}$ \\
\hline &
\end{tabular}

The proposed AM-MAC protocol is simulated based on a star network topology. The simulation area is $100 \mathrm{~m} \times 100 \mathrm{~m}$. Both IEEE802.15.4 and Wi-Fi nodes are static nodes and the number of nodes is 100. GTS is set to OFF (as only CAPCSMA/CA is assumed in the current simulation results). The proposed AM-MAC protocol uses periodic traffic with different traffic priorities that represent various QoS requirements for different smart grid applications. A selected number of nodes will generate traffic that belongs to class 1 , and some nodes will generate traffics belongs to class 2 and etc. This is required to test the AM-Mac protocol execution with different application layer QoS requirements. A constant bit rate (CBR) packets with a rate of one data packet every 5 seconds will be generated, and this traffic load will be increased in every run. Each packet has a size of 120 or 100 bytes, which corresponds to demand-side applications. However, the maximum data rate will be $100 \mathrm{kbps}$ according to HAN application communication limitations. Considering the harsh environment of SG system, the lognormal shadowing path loss model presented in equation 13 will be used. The distance between the transmitter and receiver is $\mathrm{d}$, and $\boldsymbol{d}_{\mathbf{0}}$ is the reference distance in the far-field of the transmit antenna, which is normally 8 meters. At a particular distance (d) from the transmitter, the path loss (PL) is expressed as:

$P L(d)=P L\left(d_{0}\right)+10 n \log \left(\frac{d}{d_{0}}\right)+X_{\omega}$

where $n$ is the path loss exponent, and $X_{\omega}$ represents a zero-mean Gaussian random variable with a particular standard deviation $\omega$ that is referred to as shadowing and accounts for the impact of the terrain profile on the transmit signal. The simulated indoor environment is equivalent to path loss exponent of 0.3 and shadowing deviation of 3 to represent the challenging environment presented in [35]. Three performance metrics are evaluated. First is the average packet delay, which is the average time between the generation of a packet and the reception of the corresponding acknowledgment. Second, is the packet delivery ratio (PDR), which is the ratio of the number of packets correctly acknowledged by the $\mathrm{CACo}$ and the number of packets actually that are transmitted successfully. The third is the energy consumption, which is the difference between initial node energy and current consumed energy due to packet transmission and reception. Two different scenarios are simulated to evaluate the results of the three-performance metrics for the proposed AM-MAC protocol. The first is the evaluation of the performance of AM-Mac protocol in terms of delay, PDR, and energy consumption with varying traffic loads during simulation time. The second is the evaluation of the performance with varying number of sensors.

\section{A. Packet Delay}

Fig. 4. (a) shows the performance of the average end-to-end delay. we observe that the average end to end delay increases with increasing of traffic loads in each run due to more collisions or corruption of frames due to interference, causing retransmissions required for successful data transmissions. However, the proposed AM-MAC protocol has less average delay compared to the IEEE802.15.4 MAC protocol. Fig. 4. (b) presents end to end delay which increases as the number of sensing nodes increased. The proposed AM-MAC protocol shows less end-to-end delay performance because the prioritizing operation used which enables more critical data packets with delay constrained to access medium 


\section{International Journal of Advanced Research in Computer and Communication Engineering}

Vol. 10, Issue 2, February 2021

DOI 10.17148/IJARCCE.2021.10202

immediately. On the other hand, the IEEE802.15.4 MAC protocol needs more time in retransmission operations which leads to increased packet delivery delay.

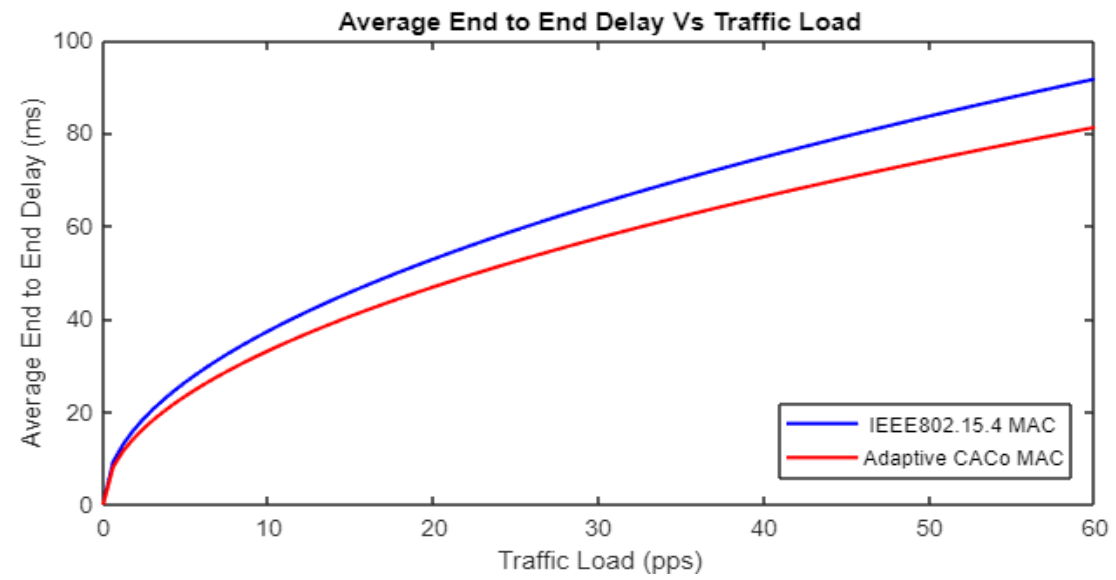

(a)

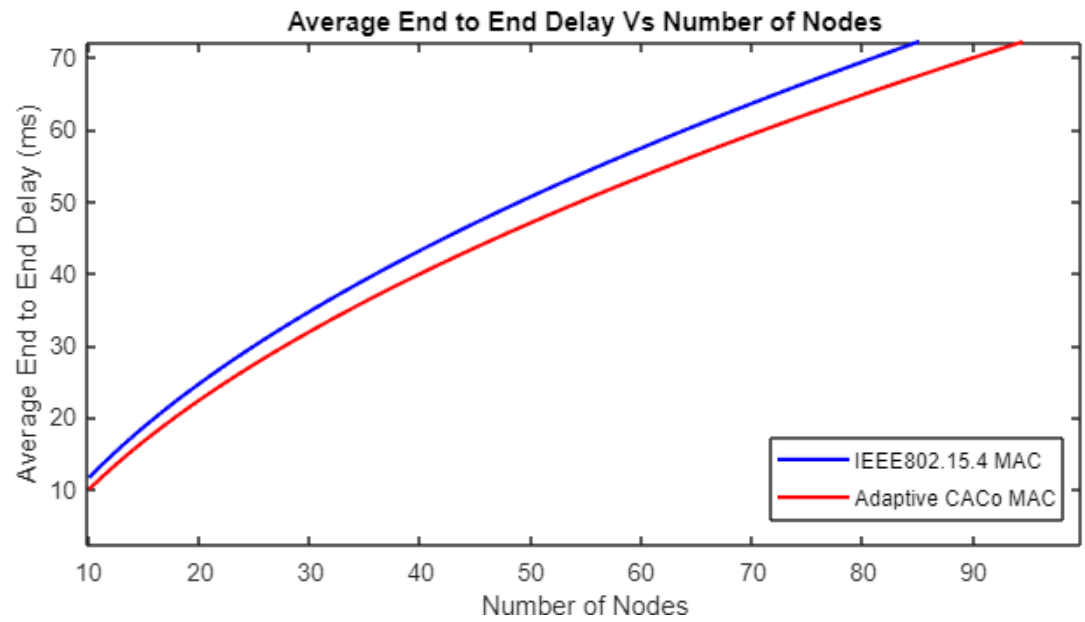

(b)

Fig. 4. Average End-to-End Delay: (a) Traffic Load (b) Number of Sensors.

B. Packet Delivery Ratio

Fig. 5. (a) shows that the average PDR decreases as the traffic load increases. The PDR rate of the proposed AM- MAC protocol is more than the IEEE802.15.4 MAC protocol. This is attributed to the interference aware algorithm that enables selection of the best channel for transmission.

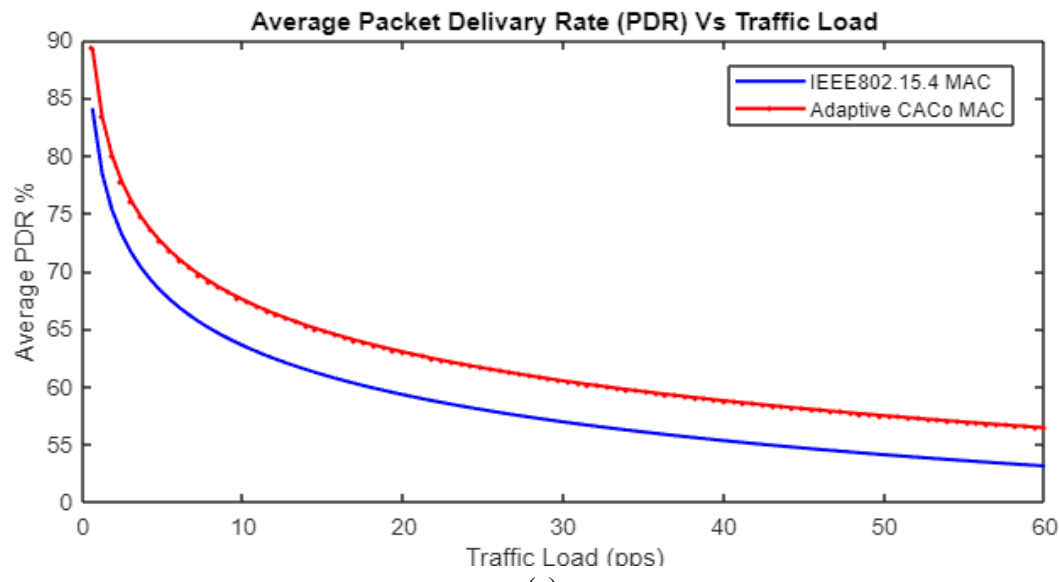

(a) 
DOI 10.17148/IJARCCE.2021.10202

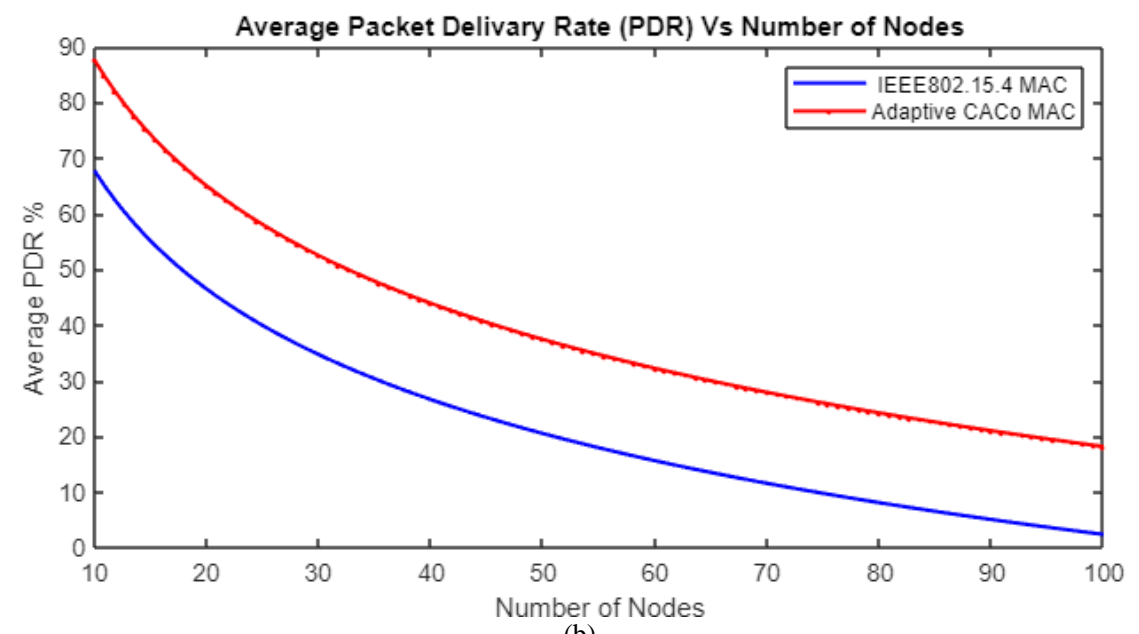

(b)

Fig. 5. Average Packet Delivery Ratio: (a) Traffic Load (b) Number of Sensors.

Fig. 5. (b) shows the average PDR decreases with the increasing number of sensor nodes and the rate of decrease in the proposed AM-MAC protocol is less than the IEEE802.15.4 MAC. The is attributed to the prioritization of data packets based on traffic class values which enable, as a result, most critical data packets to access the medium first to ensure successful transmission. As number of nodes increase, the huge number of generated packets contending for transmission cause more packets to be dropped.

\section{Energy Consumption}

Fig. 6. (a) shows that the average energy consumed increases with the increase of traffic load. The consumed energy in the proposed AM-MAC protocol is less than IEEE802.15.4 MAC due to adaptive nature of the protocol and the selection of the high-quality channels for transmissions that ensure reduced chances of interference and retransmissions. The IEEE802.15.4 MAC protocol consumes more energy because of retransmissions operations needed when interference problem accurses. Fig. 6. (b) shows that the average energy consumption increases with the increase of number of sensor nodes. The consumed energy of the proposed AM-MAC protocol is less than IEEE802.15.4 MAC due to selection of the best channel for transmissions that ensure reduced chances of interference and retransmissions.

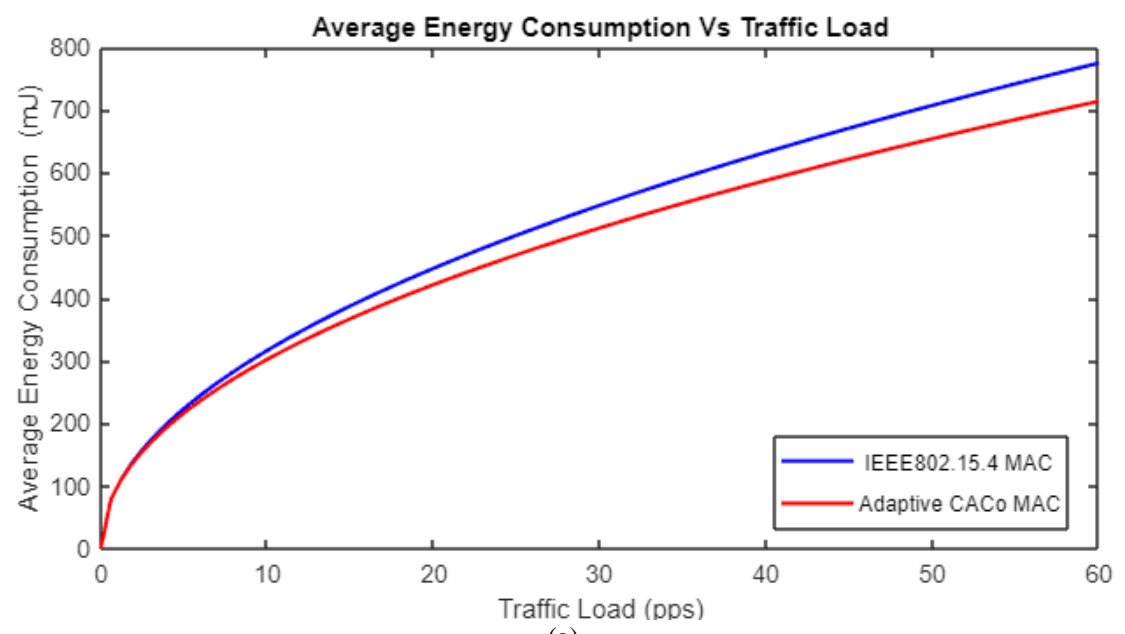

(a) 


\section{International Journal of Advanced Research in Computer and Communication Engineering}

Vol. 10, Issue 2, February 2021

DOI 10.17148/IJARCCE.2021.10202

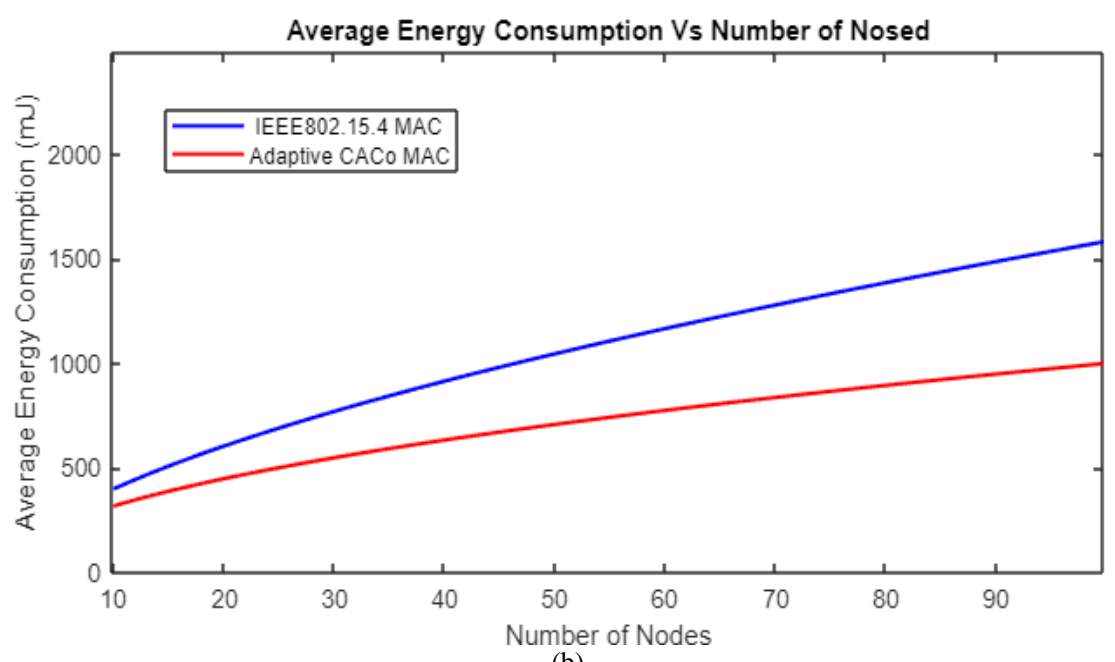

(b)

Fig. 6. Average Energy Consumption: (a) Traffic Load (b) Number of Sensors.

\section{CONCLUSION}

The simulated results show superiority of the proposed AM-MAC to mitigate interference and support more reliable and efficient communications to satisfy QoS requirements of the smart grid applications. The proposed AM-MAC was simulated and compared with the basic IEEE 802.15.4 MAC protocol. The simulated protocols were adapted to smart grid environments to ensure satisfaction of QoS requirements of various applications of WSNs. The weight cost function was formulated to solve the problem of adapting multiple conflicting parameters. The results have demonstrated that the proposed AM-MAC protocol was effective to mitigate interference, satisfy QoS requirements, and maintain the energy efficiency of WSN. One of the limitations of the proposed algorithm is that it needs prior communications on each channel to collect enough information about the three parameters used in the proposed MAC. The dependence on some historical data may not work well for the dynamic smart grid environments. Therefore, an adaptive channel selection algorithm to select channels immediately when interference increases during a transmission is needed, instead of continuing the transmission with corrupted data. In addition, channels with good quality may be accidentally interfered with any other channel and thus a periodic evaluation is necessary.

\section{REFERENCES}

[1]. L. Chhaya, P. Sharma, G. Bhagwatikar, and A. Kumar, "Wireless sensor network based smart grid communications: cyber attacks, intrusion detection system and topology control," Electronics, vol. 6, p. 5, 2017.

[2]. J. Nassar, M. Berthomé, J. Dubrulle, N. Gouvy, N. Mitton, and B. Quoitin, "Multiple Instances QoS Routing in RPL: Application to Smart Grids," Sensors, vol. 18, p. 2472, 2018.

[3]. M. Centenaro, L. Vangelista, A. Zanella, and M. Zorzi, "Long-range communications in unlicensed bands: The rising stars in the IoT and smart city scenarios," IEEE Wireless Communications, vol. 23, pp. 60-67, 2016.

[4]. P. Yi, A. Iwayemi, and C. Zhou, "Developing ZigBee deployment guideline under WiFi interference for smart grid applications," IEEE transactions on smart grid, vol. 2, pp. 110-120, 2011.

[5]. P. H. Gomes, Y. Chen, T. Watteyne, and B. Krishnamachari, "Insights into frequency diversity from measurements on an indoor low power wireless network testbed," in 2016 IEEE Globecom Workshops (GC Wkshps), 2016, pp. 1-6.

[6]. M. Hassan, L. Kamarudin, and A. Zakaria, "Mitigating interference in a heterogeneous wireless network using channel selection," in Special Issue for International Conference of Advanced Materials Engineering and Technology, 2013.

[7]. X. Chen, J. Fan, K. Cheng, and M. Chen, "Analysis of Anti Interference of Channel Control Measure in $2.4 \mathrm{GHz}-$ Band Network," in International Conference on Mechatronics and Intelligent Robotics, 2017, pp. 83-92.

[8]. M. Talebi, C. Papatsimpa, and J.-P. M. Linnartz, "Dynamic performance analysis of IEEE 802.15. 4 networks under intermittent Wi-Fi interference," in 2018 IEEE 29th Annual International Symposium on Personal, Indoor and Mobile Radio Communications (PIMRC), 2018, pp. $1-7$.

[9]. P. Yi, A. Iwayemi, and C. Zhou, "Frequency agility in a ZigBee network for smart grid application," in 2010 Innovative Smart Grid Technologies (ISGT), 2010, pp. 1-6.

[10]. Q. Yang, J. A. Barria, and T. C. Green, "Communication infrastructures for distributed control of power distribution networks," IEEE Transactions on Industrial Informatics, vol. 7, pp. 316-327, 2011.

[11]. K. Moslehi and R. Kumar, "Smart grid-a reliability perspective," in Innovative Smart Grid Technologies (ISGT), 2010, 2010, pp. 1-8.

[12]. T. Lindner, D. Wyrwał, and A. Kubacki, "Low Power Wireless Protocol for IoT Appliances Using CSMA/CA Mechanism," in Conference on Automation, 2019, pp. 199-207.

[13]. I. S. 802.15.4a ${ }^{\mathrm{TM}}-2007$, "IEEE802.15.4 standred" 31 August 2007.

[14]. B. C. Villaverde, S. Rea, and D. Pesch, "Guaranteeing reliable communications in mesh beacon-enabled IEEE802. 15.4 WSN for industrial monitoring applications," in International Conference on Ad Hoc Networks, 2010, pp. 359-370. 


\section{International Journal of Advanced Research in Computer and Communication Engineering}

Vol. 10, Issue 2, February 2021

\section{DOI 10.17148/IJARCCE.2021.10202}

[15]. D. Bhaskar and B. Mallick, "Performance Evaluation Of MAC Protocol For IEEE 802.11, 802.11 Ext. WLAN And IEEE 802.15. 4 WPAN Using NS-2," International Journal of Computer Applications, vol. 119, pp. 21153-4151, 2015.

[16]. C. Wang, T. Jiang, and Q. Zhang, ZigBee® network protocols and applications: CRC Press, 2014.

[17]. S. Bhandari and S. Moh, "A survey of MAC protocols for cognitive radio body area networks," Sensors, vol. 15, pp. 9189-9209, 2015.

[18]. K. B. Letaief and W. Zhang, "Cooperative Communications for Cognitive Radio Networks," Proceedings of the IEEE, vol. 97, pp. 878-893, 2009.

[19]. O. B. Akan, O. B. Karli, and O. Ergul, "Cognitive radio sensor networks," IEEE network, vol. 23, pp. 34-40, 2009.

[20]. A. Ahmad, S. Ahmad, M. H. Rehmani, and N. U. Hassan, "A survey on radio resource allocation in cognitive radio sensor networks," IEEE Communications Surveys \& Tutorials, vol. 17, pp. 888-917, 2015.

[21]. A. Koubaa, M. Alves, and E. Tovar, "A comprehensive simulation study of slotted CSMA/CA for IEEE 802.15. 4 wireless sensor networks," in 2006 IEEE international workshop on factory communication systems, 2006, pp. 183-192.

[22]. Y. Ren, S. He, P. Hu, and J. Sun, "Research on Cross-Layer Optimization of Smart Grid Demand Side Based on WSNs," pp. 414-420, 2018.

[23]. L. Nassef, R. Elhebshi, and L. Jose, "Evaluating Performance of Wireless Sensor Network in Realistic Smart Grid Environment," International Journal of Wireless \& Mobile Networks (IJWMN) Vol, vol. 10, 2018.

[24]. M. F. Khan, E. A. Felemban, S. Qaisar, and S. Ali, "Performance analysis on packet delivery ratio and end-to-end delay of different network topologies in wireless sensor networks (WSNs)," in 2013 IEEE 9th International Conference on Mobile Ad-hoc and Sensor Networks, 2013, pp. 324-329.

[25]. T. Watteyne, J. Weiss, L. Doherty, and J. Simon, "Industrial IEEE802. 15.4 e networks: Performance and trade-offs," in 2015 IEEE International Conference on Communications (ICC), 2015, pp. 604-609.

[26]. E. Kabalci and Y. Kabalci, Smart Grids and Their Communication Systems: Springer, 2019.

[27]. T. Kaur and D. Kumar, "QoS mechanisms for MAC protocols in wireless sensor networks: a survey," IET Communications, 2019.

[28]. Z. Chen, Y. Liu, R. Liu, J. Yuan, and D. Han, "Improved CSMA/CA Algorithm Based on Alternative Channel of Power Line and Wireless and First-Time Idle First Acquisition," IEEE Access, vol. 7, pp. 41380-41394, 2019.

[29]. H. Zhao, M. Qiu, K. Gai, and X. He, "Optimal solution to intelligent multi-channel wireless communications using dynamic programming," The Journal of Supercomputing, vol. 75, pp. 1894-1908, 2019.

[30]. E. De Poorter, J. Hoebeke, M. Strobbe, I. Moerman, S. Latré, M. Weyn, et al., "Sub-GHz LPWAN network coexistence, management and virtualization: an overview and open research challenges," Wireless Personal Communications, vol. 95, pp. 187-213, 2017.

[31]. M. Yigit, O. Durmaz Incel, S. Baktir, and V. C. Gungor, "QoS - Aware MAC protocols utilizing sectored antenna for wireless sensor networks based smart grid applications," International Journal of Communication Systems, vol. 30, p. e3168, 2017.

[32]. B. Nahar, M. S. Alam, S. A. Shawkat, and M. A. Hoque, "Priority Aware Interference Mitigation Techniques for Coexistence of Wireless Technologies in Smart Utility Networks," in Proceedings of the 13th ACM Symposium on QoS and Security for Wireless and Mobile Networks, 2017, pp. 105-112.

[33]. A. Köpke, M. Swigulski, K. Wessel, D. Willkomm, P. Haneveld, T. E. Parker, et al., "Simulating wireless and mobile networks in OMNeT++ the MiXiM vision," in Proceedings of the 1st international conference on Simulation tools and techniques for communications, networks and systems \& workshops, 2008, p. 71.

[34]. J. Marinho and E. Monteiro, "Cognitive radio simulation based on omnet++/mixim," in Proc. of the 11th Conferencia sobre Redes de Computadores, 2011.

[35]. V. C. Gungor, B. Lu, and G. P. Hancke, "Opportunities and challenges of wireless sensor networks in smart grid," IEEE transactions on industrial electronics, vol. 57, pp. 3557-3564, 2010. 